\title{
Photometric Variability of Helium Stars in the Z-bump Instability Strip
}

\author{
R. Aznar Cuadrado, P. Montañés Rodríguez, C. S. Jeffery \\ Armagh Observatory, College Hill, Armagh BT61 9DG, N. Ireland \\ D. Pollacco \\ Isaac Newton Group, Santa Cruz de La Palma, Tenerife 38780, Canary \\ Islands, Spain
}

\begin{abstract}
Z-bump opacities are known to excite radial pulsations in helium stars with $10^{2} \lesssim L / M \lesssim 10^{4}$ and $T_{\text {eff }} \sim 25000 \mathrm{~K}$. Well-studied cases are $\mathrm{V} 652 \mathrm{Her}$ and $\mathrm{BX}$ Cir. The absence of variations in the metalpoor star HD 144941 demonstrates the importance of Z-bump opacities to drive these pulsations. Several other helium-rich stars lie close to the theoretical blue-edge of the Z-bump instability strip. One of these, LS IV $+6^{\circ} 2$, is a known radial velocity variable. We report on recent (1999 June) observations of two helium stars close to the Z-bump instability boundary. Both appear to be variable.
\end{abstract}

\section{Introduction}

Stellar pulsations can arise when a local maximum in opacity is situated appropriately in a stellar envelope, giving rise to several instability strips in the HR diagram, most notably the Cepheid strip. Pulsations driven by iron-group opacities and by CNO-opacities occur respectively in B-type stars (e.g. $\beta$ Cephei stars) and very hot subdwarfs (PG 1159 stars). The excitation of such pulsations, radial or nonradial, is a sensitive function of chemical composition and often requires the enhancement of some driving species (e.g. iron) or the suppression of damping species (e.g. hydrogen).

The carbon-rich helium star LS IV $+6^{\circ} 2$ (Jeffery 1998) was suspected to be variable, although it lies outside the Z-bump instability finger (see Jeffery \& Saio 2000). Another helium star, LSS5121 (Heber, Jonas, \& Drilling 1985), probably resembles the more luminous helium star V2076 Oph, which lies above the horizontal part of the instability boundary (Saio \& Jeffery 1988) and pulsates nonradially (Lynas-Gray et al. 1987). Periods of the order of $0.05 \mathrm{~d}^{-1}$ and $0.1 \mathrm{~d}^{-1}$ were predicted for LS IV $+6^{\circ} 2$ and LSS5121 respectively, assuming a hypothetical mass of $0.5 M_{\odot}$ (Jeffery \& Saio 1999).

In this paper we describe preliminary results from new photometric observations of these helium stars, LS IV $+6^{\circ} 2$ and LSS5121, which have been obtained in order to explore observationally the blue boundary of the Z-bump instability finger. 


\section{Observations, Reduction and Results}

CCD-imaging photometric observations of extreme helium stars LS IV $+6^{\circ} 2$ and LSS5121 were obtained with the 1-m Jacobus Kapteyn Telescope at the Roque de los Muchachos Observatory, La Palma. Strömgren filters $u, b, v, y$ were used. Observations were performed on the nights of $1999 \mathrm{June} 3,5,6$, and 7 . The detector used at the telescope was TEK4 with $1024 \times 102424 \mu \mathrm{m}$ pixels. For TEK4 the image scale is $0.33 \mathrm{arcsec} /$ pixel, giving a field of view of $5.6 \times 5.6$ arcmin. Exposure times were 70 and $100 \mathrm{~s}$ in $y$ for LS IV $+6^{\circ} 2$ and LSS5121 respectively. Standard stars were chosen in order to cover a large range in $b-y$. Secondary Strömgren standards were selected from Stetson (1991). Data reduction was done using IRAF. Each frame was corrected for bias and flat-field. Automatic aperture photometry was used to obtain instrumental magnitudes for many stars in each frame. Simultaneous differential Strömgren $y$ photometry was obtained by using comparison stars from the same CCD frames. In order to avoid the possibility of choosing a variable star as a comparison, we calculated the difference in magnitudes of our objects with as many comparison stars as we could find in the frames. In this way we could eliminate the possibility that any detected variations were due to some particular comparison star. At this preliminary stage of the data reductions, our results are more exploratory than definitive. Data from three other filters and additional comparison stars will be available in due course.

From observations of June 5 we might infer a period of a few hours for LS IV $+6^{\circ} 2$. Data from other nights also show some variability, but no single period is evident. Consequently we made a search for periodic behaviour by calculating a periodogram (Scargle 1982) for the complete dataset. Although strongly biased by the $1 \mathrm{~d}^{-1}$ aliases, there is tentative evidence for periodicity around $5 \mathrm{~d}^{-1}$; other frequencies, or none, may be present.

The available data for LSS5121 do not show any strongly marked trend, although a first impression from the light curves suggests that this star is also variable. There are no significant peaks in the periodogram. The lack of more complete photometric coverage of LSS5121 is due to the fact that during our observing run LSS5121 could be observed for less than four hours each night.

\section{References}

Heber, U., Jonas, G., \& Drilling, J. S. 1986, in Proc. IAU Coll. 87, HydrogenDeficient Stars and Related Objects, ed. K. Hunger, D. Schoenberner, \& N. K. Rao (Dordrecht: Reidel), 67

Jeffery, C. S. 1998, MNRAS, 294, 391

Jeffery, C. S. \& Saio, H. 1999, MNRAS (in press)

Jeffery, C. S. \& Saio, H. 2000, in these proceedings, p. 440

Lynas-Gray, A. E., Kilkenny, D., Skillen, I., \& Jeffery, C. S. 1987, MNRAS, 227, 1073

Saio, H. \& Jeffery, C. S. 1988, ApJ, 328, 714

Scargle, J. D. 1982, ApJ, 263, 835

Stetson, P. B. 1991, AJ, 102, 589 